\title{
Persistence of the Polarization in a Fusion Process
}

\author{
J.-P. Didelez ${ }^{1, a}$ and C. Deutsch ${ }^{2}$ \\ 1 Institut de Physique Nucléaire, CNRS/IN2P3 \& Université Paris XI 91406 Orsay, France \\ 2 Laboratoire de Physique des Gaz et des Plasmas, CNRS \& Université Paris XI, 91405 Orsay, France
}

\begin{abstract}
We propose an experiment to test the persistence of the polarization in a fusion process, using a petawatt laser hitting a polarized $H D$ target. The polarized protons and deuterons heated in the plasma induced by the laser have a significant probability to fuse producing a ${ }^{3} \mathrm{He}$ and a $\gamma$ ray or a neutron in the final state. The angular distribution of the radiated $\gamma$ rays and the change in the correponding total cross section are related to the polarization persistence, but the resulting signal turns out to be weak. By comparison, the neutrons are produced hadronically with a larger cross section and are much easier to detect experimentally. A significant reduction of the cross section by parallel polarization of the deuterons is reliably predicted by the theory. Therefore, it is expected that the corresponding signal on the neutron counting rate could be seen experimentally.
\end{abstract}

\section{Introduction}

The polarization of $D$ and $T$ nuclei should increase their reactivity when used as fuel material in fusion processes induced either by magnetic or by inertial confinement.

The fusion reaction:

$$
D+T \rightarrow \alpha+\text { neutron }+17.6 \mathrm{MeV}
$$

goes mainly hrough the excitation of an ${ }^{5} \mathrm{He} 3 / 2^{+}$intermediate state, resulting from the coupling of the spins 1 and $1 / 2$ of the $D$ and $T$ nuclei to a total spin $S=3 / 2$. Without polarization of $D$ and $T$, the statistical distribution of the six possible states gives four $\mathrm{S}=3 / 2$ and two $\mathrm{S}$ $=1 / 2$ states. Only the $3 / 2$ states can produce the intermediate $3 / 2$ resonance. With $100 \%$ parallel polarization of $D$ and $T$, all states would contribute to the fusion, increasing the reactivity by $50 \%$. In addition, the polarization allows to control the direction in which the reaction products are emitted, in particular the neutrons have a $\sin ^{2} \theta$ distribution. This can be very useful to reduce damages or activation of costly equipments [1]. The question is to know if the polarization will persist in a fusion plasma. We propose to investigate the polarization persistency using the reactions:

$$
\begin{gathered}
P+D \rightarrow^{3} H_{e}+\gamma+5.5 \mathrm{MeV} \\
D+D \rightarrow^{3} H_{e}+n+3.267 \mathrm{MeV}
\end{gathered}
$$

induced by fusion of polarized protons and deuterons heated in a plasma. It is anticipated that the angular distribution of the radiated $\gamma$ rays or significant changes in the fusion rates can be related to the persistence of the polarization.

\section{Magnetic versus Inertial confinement}

The idea of inertial confinement is to compress tiny amounts of DT - simultaneously with heating - to such an extent that sufficient fuel burn is achieved within the time interval the fuel keeps together inertially. It turns out that the plasma density $\mathrm{n}$ and confinement time $\tau$ required for inertial fusion are very different from those for manetic fusion (11 orders of magnitude):

$\begin{array}{lllc}\text { confinement } & \mathrm{n}\left(\mathrm{cm}^{-3}\right) & \tau(\mathrm{sec}) & \mathrm{n} . \tau\left(\mathrm{sec} / \mathrm{cm}^{3}\right) \\ \text { magnetic } & 10^{14} & 10 & 10^{15} \\ \text { Inertial } & 10^{26} & 10^{-10} & 10^{16}\end{array}$

In both cases, however, the product $n . \tau$ has to satisfy the Lawson criterion (n. $\tau \geq 10^{15} \mathrm{sec} / \mathrm{cm}^{3}$ ) which is set by the $D T$ fusion physics.

In a Tokamak like ITER, the confinement time is expected to be as large as $300 \mathrm{~s}$, which makes it very difficult for the polarization to survive till the end of the cycle, while at MEGAJOULE, the whole compression time of a tiny target is of the order of 35 ns, making it much easier for the survival of the polarization. Kulsrud [1] has investigated several depolarization mechanisms as: 1) inhomogeneous static magnetic fields, 2) binary collisions, 3) magnetic fluctuations, 4) atomic effects, and concluded that all of them are weak. Relaxation times can become very long, when the depolarization paths are suppressed, as for example for $H D$ [2]. However, in this matter, an experimental verification is always needed. In US, there is a project [3] to inject polarized $\mathrm{D}$ and ${ }^{3} \mathrm{He}$ in the DIII-D tokamak of San Diego, in order to see a $15 \%$ increase of the reaction rate of emitted protons by the fusion reaction:

$$
D+{ }^{3} H_{e} \rightarrow{ }^{4} H_{e}+p+18.35 \mathrm{MeV}
$$




\section{Polarized HD Target $25 \mathrm{~cm}^{3}$$$
\mathrm{H}(\mathrm{p}) \text { polarization }>60 \%
$$$$
\text { D (d) vect. polar. }>14 \%
$$

5.5 MeV y ray from

$\mathrm{p}+\mathrm{d} \rightarrow{ }^{3} \mathrm{He}+\mathrm{y}$

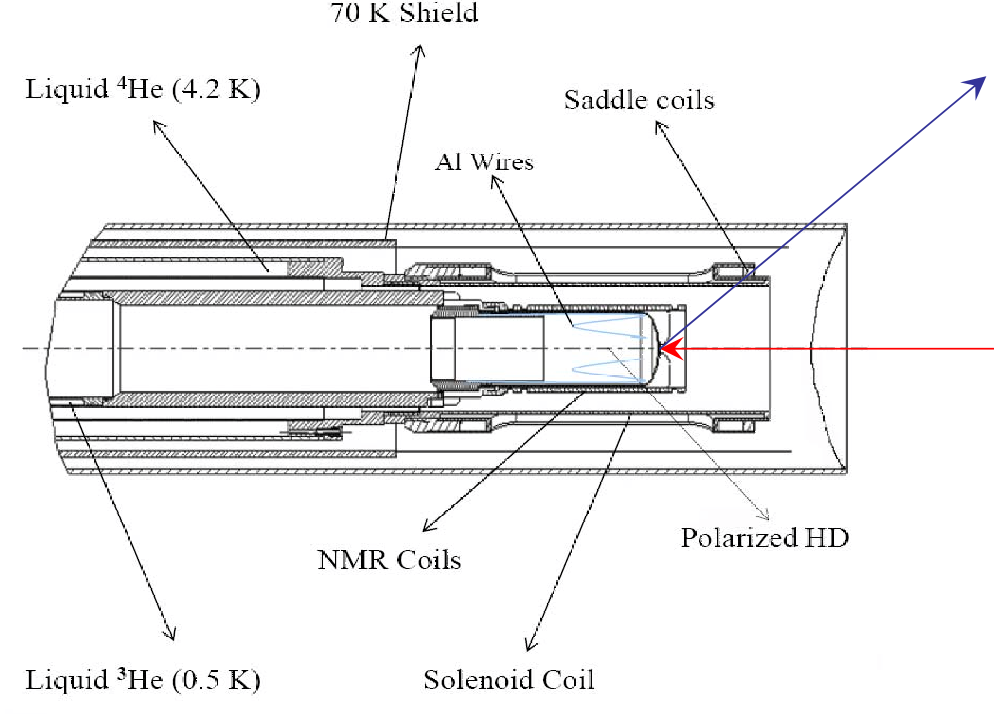

2.45 MeV $\mathrm{n}$ from

$\mathrm{d}+\mathrm{d} \rightarrow{ }^{3} \mathrm{He}+\mathrm{n}$

Powerful Laser (Petawatt)

creates a local plasma

of $p$ and $d$ ions ( $5 \mathrm{KeV}$ )

\section{$200 \mathrm{~mJ}, 160 \mathrm{fs}$ \\ $4.5 \mu \mathrm{m}$ FWHM \\ $790 \mathrm{~nm}, \sim 10^{18} \mathrm{~W} / \mathrm{cm}^{2}$}

Fig. 1. Tentative set-up showing a typical arrangement of a polarized $H D$ target in a cryostat maintaining the target temperature below $1 \mathrm{~K}$ under a holding field of $1 \mathrm{~T}$. The target is bombarded by a petawatt laser producing a localized plasma. Neutrons and $\gamma$ rays are produced in the plasma, by fusion reactions between polarized protons and deuterons.

However, the injection of $55 \%$ polarized $D$ and ${ }^{3} H_{e}$ into a tokamak is a problem in itsef, requiring technical innovations which may take some time.

\section{Tentative set-up}

At IPN Orsay, we have developed the static polarization of $H D$ molecules for samples as large as $25 \mathrm{~cm} 3$ [4]. It has been demonstrated, that the distillation and the ageing technique [5] allow to get nuclear relaxation times larger than one week, even at $1.5 \mathrm{~K}$ and $1 \mathrm{~T}$. Proton polarization in excess of $60 \%$ and deuteron vector polarization higher than $14 \%$ have been achieved. It is advocated that a petawatt laser hitting a piece of polarized $H D$ ice will induce locally a plasma hot enough to allow the fusion reactions (2) and (3) to take place and to be measured. If both $H$ and $D$, namely the proton and the deuteron of the $H D$ molecules are polarized in the same direction and have kept their polarization in the fusion process, the $5.5 \mathrm{Mev} \gamma$ ray will be emitted with some angular distribution relative to the polarization axis, also the fusion rates will depend drastically on the initial state polarizations. A tentative sketch of the experimnetal set-up is displayed in Fig. 1. It should be mentionned that with a power of $200 \mathrm{~mJ} / \mathrm{shot}$, the laser repetition rate can be adjusted to prevent melting of the target. Without cooling power provided by the holding cryostat,
1,000 such laser shots would be necessary to melt completely $25 \mathrm{~cm}^{3}$ of solid $H D$. The overall polarization will decrease with time, but is continuously monitored by the NMR coils.

Back in 1970, a french group of the "Commissariat à l'Énergie Atomique" in France [6] reported the observation of neutron emission from DD fusion, after focussing a $3 \mathrm{GW}$ fast laser on a piece of $D_{2}$ ice $1 \mathrm{~mm}^{2}$ in cross section. At that time, a rise time of $5 \mathrm{~ns}$ was considered as fast. Since then Petawatt lasers have been developped using the chirped pulse amplification, able to deliver several tens of J within 20 fs to 1 ps. Those lasers can be used for fast ignition in inertial confinement fusion [7] or to accelerate particles [8].

Pretzler [9] reports quantitative data resulting from the irradiation of $C_{2} D_{4}$ targets with laser pulses $(200 \mathrm{~mJ}, 160$ fs, $4.5 \mu \mathrm{m}$ FWHM, $790 \mathrm{~nm}, \sim 10^{18} \mathrm{~W} / \mathrm{cm}^{2}, 10 \mathrm{~Hz}$ ). A total rate of 140 neutrons per shot could be produced, through the fusion reaction (3).

The technique to produce ion beams with ultra high power lasers starts to be well documented [10]: scaling laws and models exist which can predict the number and energy distribution of accelerated particles. This in turn, allows to tentatively optimize experimental conditions, although in this field, large uncertainties remain concerning the details of the processes. In particular, it is not at all sure that in a block of ice, ion acceleration will take place: 


\section{$19^{\text {th }}$ International IUPAP Conference on Few-Body Problems in Physics}

acceleration of particles have been reported only with thin targets.

From the quantitative data of Ref. [9], given the measured cross sections of reaction (2): $\sigma_{0}(10 \mathrm{keV})=18 \mu \mathrm{b}$ [11]; $\sigma_{0}(10 \mathrm{MeV})=1 \mathrm{mb}$ [12], $1-10$ (radiative captures / laser shot) could be expected. The detection of the correponding $\gamma$ rays is a serious experimental problem. Conventional Ge detectors cannot be used, because of the large number of energetic electrons and $\gamma$ rays emitted in an extremely short time, which will pile-up in the Ge detector. Pair spectrometers would perform better, in spite of their lower efficiency.

\section{The "Few-Body" problems}

For the radiative capture (2), the experiment is essentially based on the angular distribution of the radiated $\gamma$ ray with respect to the polarization axis. Assuming that $P$ and $D$ nuclei collide from all directions in a hot plasma, with a total spin $\mathrm{S}=3 / 2$ (quartet transitions: $\sigma_{4}$, namely $100 \%$ polarization), while an unpolarized plasma involves also transitions from a total spin $S=1 / 2$, (doublet transitions: $\left.\sigma_{2}\right)$, the angular dependence has the form [13]:

$$
d \sigma_{4} / d \omega \sim\left(1+\cos ^{2} \theta\right)
$$

At the energies of interest, tens of $\mathrm{keV}$ or so, the process proceeds via $\mathrm{S}$ and $\mathrm{P}$ wave capture, and is induced predominantly by magnetic (for S-wave) and electric (for P-wave) dipole transitions. Higher multipoles, at the low energies considered here, can be neglected [14]. In addition, the p-wave contributions are much smaller than the $\mathrm{S}$-wave one, since they involve the small D-wave component of the ${ }^{3} H_{e}$ bound state. Therefore, although P-wave contributions involve isotropic and $\left(3 \times \cos ^{2} \theta-1\right)$ terms, one would expect a very small distortion of the $\left(1+\cos ^{2} \theta\right)$ angular distribution due to a pure $\mathrm{S}$-wave magnetic dipole transition, as given by Eq. (5). From experimental point of view, this angular distribution means that $1 / 3$ of the $\gamma$ rays due to quartet transitions will be preferentially emitted in the direction of polarization, namely in the direction of the laser beam. However, it is out of question to put a $\gamma$ ray detector in the direction of the laser beam, because of the large number of energetic electrons produced at forward angles. A transverse position, typically 90 degrees, is the most convenient. There the effect of the polarization on the quartet $\gamma$ rays counting rate is reduced to $25 \%$ to be compared to values approaching $100 \%$ at forward angles. Taking into account the highest acheavable $P$ and $D$ polarization rates of respectively $80 \%$ and $30 \%$ in $H D$ by the static polarization method and the dominant $\gamma$ ray contribution coming from doublet transitions for unpolarized nuclei: typically $\sigma_{4} / \sigma_{\text {unpol }} \simeq 0.2$ from theoretical estimates [13] and even much smaller from experimental results at low energies [15], one cannot expect a signal larger than $3 \%$ on the counting rates between polarized and unpolarized targets. This makes the radiative capture experiment fairly difficult to exploit. It should be mentionned here, that the $H D$ polarization technology allows to polarize $H$ and $D$ in an antiparallel configuration [16] in order to enhance the dominant $\sigma_{2}$ contribution. So doing, an increase $\sigma_{\text {pol }} / \sigma_{\text {unpol }} \simeq 1.07$, namely $7 \%$ could be expected and eventually measured.

There is an alternative possibility offered by the hadronic fusion reaction (3) producing $2.45 \mathrm{MeV}$ neutrons which are much easier to detect than $\gamma$ rays in a surrounding background. Based on a partial wave analysis [17], it has been argued that the cross section should be significantly reduced if the interacting deuterons have parallel vector polarizations (i.e. with total $\operatorname{spin} \mathrm{S}=2$, namely quintet transitions: $\sigma_{5}$ ) [1], but resonating-group calculations [18] found that polarized fusions are not suppressed. However, it is known that resonating-group calculations are not very reliable for weekly bound nuclei as deuterons [19]. On the other hand, DWBA calculations give a large Quintet Suppression Factor (QSF): $\sigma_{5} / \sigma_{\text {unpol }} \simeq 0.08$ in the range $E_{d}=$ $20-150 \mathrm{keV}$ [20]. Large reduction factors are confirmed by recent calculations [21], with QSF going from 0.5 at $100 \mathrm{KeV}$ to 0.2 at $4 \mathrm{MeV}$. In addition total cross sections are in the range of $100 \mathrm{mb}$ [22], to be compared to $100 \mu \mathrm{b}$ for the electromagnetic reaction (2) [11]. In view of those considerations, the $D+D \rightarrow^{3} H_{e}+n$ is the way to go. It should be noted that for a polarized $H D$ target, it is possible to increase the $D$ polarization above $50 \%$ at the expense of the $P$ one, by transfer of the $P$ polarization to $D$, using adiabatic fast passage [16]. A decrease of the emitted neutron counting rate of 10-20\% going from a unpolarized target to a polarized one, namely $\sigma_{\text {pol }} / \sigma_{\text {unpol }} \simeq 0.85$ should be easily measurable. The corresponding effect is further increased by the fact that the neutrons produced by quintet transitions are preferentially emitted perpendicular to the polarization axis [22].

\section{CONCLUSION}

A considerable effort in under way to produce energy using controlled fusion either by magnetic or by inertial confinement. Polarized fusion fuel is of great interest, both to increase the fuel reactivity and to control the direction in which the reaction products are emitted. The question is to know if the polarization will persist in a fusion process. We propose a possibility to investigate this point using high power laser beams on polarized $H D$ samples through fusion reactions like: $P+D \rightarrow{ }^{3} H_{e}+\gamma$ or $D+D \rightarrow{ }^{3} H_{e}+n$. Before undertaking the corresponding experimental venture, precise predictions of the cross sections and polarization observables at low and moderate energies were needed, which is precisely in the scope of the present Few-Body Conference. Those predictions were kindly and rapidly performed by the relevant specialists after the FB19 meeting. It turns out that the radiative capture, which was initialy considered to demonstrate the persistence of the polarization in a fusion process is not the prefered way to go, because the $\gamma$ rays, not only are difficult to select, but they are emitted preferentially along the polarization axis, in a region of high electromagnetic background. In addition, the low cross sections attached to an electromagnetic process, make it very difficult to pin down a signal smaller than $3 \%$ on the couting rates, although signifcant change in the total 
cross section ( $7 \%$ ) could be exploited in a different polarization scheme: $\mathrm{P}$ and $\mathrm{D}$ in an antiparallel configuration.

By comparison, the hadronic fusion seems much better, having a cross section larger by 3 orders of magnitude and producing a signal as large as $10-20 \%$ on the neutron counting rates, further increased by a favorable angular distribution of the neutrons emitted by quintet transitions. Neutron counters can be shielded and can work in a high background environment. Polarized target preparation is more difficult, requiring high deuteron polarization, but the relevant techniques are now well established.

\section{Acknowledgements:}

It is a pleasure to acknowledge Pr. Eduard A. Kuraev for having derived "on the back of an envelope" the angular distribution of radiative captures, during his visiting time at IPN Orsay. We are also grateful to our colleague Dr. Egle Tomasi-Gustafson for many discussions and for having brought to our attention her work on the subject [23]. Finally, definite conclusions would not have been possible without the calculations of Pr. R. Schiavilla, Dr. M. Viviani and Dr. A. Deltuva, who promptly after the Bonn FB19 Conference, derived the relevant fusion observables, using their most recent theoretical approaches.

\section{References}

1. R. M. Kulsrud et al., Phys. Rev. Lett. 49, 1248 (1982).

2. A. Honig, Phys. Rev. Lett. 19, 1009 (1967).

3. A. Honig, Private communication.

4. S. Bouchigny et al., Distillation and Polarization of $H D$, in: T. Uesaka, H. Sakai, A. Yoshimi and K. Asahi (Eds.), Proceedings of the PST05 Workshop, Tokyo, Japan, World Scientific, Singapore, November 2005, p. 67.

5. S. Bouchigny et al., Nucl. Inst. and Meth. A 607, 271 (2009) and references therein.

6. F. Floux et al., Phys. Rev. A 1, 821 (1970).

7. M. Zepf et al., Phys. Plasmas 3, 3242 (1996).

8. R.A. Snavely et al., Phys. Rev. Lett. 85, 2945 (2000).

9. G. Pretzler et al., Phys. Rev. E 58, 1165 (1998).

10. M. Schollmeier et al., Nucl. Inst. and Meth. 577, 186 (2007) and references therein.

11. G. J. Schmid et al., Phys. Rev. C 52, R1732 (1995).

12. D. M. Skopic et al., Phys. Rev. C 19, 601 (1979).

13. M. Viviani, Private communication.

14. M. Viviani et al., Phys. Rev. C 54, 534 (1996); ibid. C 61, 064001 (2000).

15. M. Konijnenberg, $P h D$ Thesis, Technical University Delft, 1990, ECN-R-90-1 Report.

16. J.-P. Didelez, Nucl. Phys. News 4, 10 (1994).

17. B. P. Ad'yasevich et al., Sov. J. Nucl. Phys. 9, 167 (1969); ibid. 33, 601 (1981).

18. H. M. Hofmann and D. Fick, Phys. Rev. Lett. 52, 2033 (1984).

19. S. Quaglioni, "Scattering of light nuclei", This Conference.

20. J. S. Zhang et al., Phys. Rev. Lett. 55, 1649 (1985); ibid. 57, 1410 (1986).
21. A. Deltuva and A. C. Fonseca, Phys. Rev. C 76, 021001(R) (2007); A. Deltuva et al., Phys. Lett. B 660, 471 (2008).

22. A. Deltuva, Private communication.

23. M. P. Rekalo and E. Tomasi-Gustafson, "Nuclear processes in magnetic fusion reactors with polarized fuel", arXiv:nucl-th/0010046v1, 16 oct 2000. 